\title{
A Framework for Bottom Up Specialisation of Logic Programs
}

\author{
Wim Vanhoof ${ }^{\star}$ Danny De Schreye ${ }^{\star \star}$ Bern Martens ${ }^{\star \star \star}$ \\ Department of Computer Science, Katholieke Universiteit Leuven, \\ Celestijnenlaan 200A, B-3001, Heverlee, Belgium. \\ Tel: ++3216327638 Fax: ++3216327996 \\ e-mail: \{wimvh, dannyd, bern\}@cs.kuleuven.ac.be
}

\begin{abstract}
In this paper, we develop a solid theoretical foundation for a bottom up program transformation, capable of specialising a logic program with respect to a set of unit clauses. Extending a well-known operator, originally introduced for composing logic programs, we define a bottom up partial deduction operator and prove correctness of the transformation with respect to the S-semantics. We also show how, within this framework, a concrete control strategy can be designed.

The transformation can be used as a stand-alone specialisation technique, useful when a program needs to be specialised w.r.t. its internal structure (e.g. a library of predicates w.r.t. an abstract data type) instead of a goal. On the other hand, the bottom up transformation can be combined with a more traditional top down partial deduction strategy. We conjecture that such a combined approach will finally enable good automatic specialisation of meta-programs.
\end{abstract}

\section{Introduction}

Partial deduction is an important transformation technique for logic programs, capable of removing substantial inefficiencies from programs $[16,9,5]$. As an online specialisation technique, it is based on an evaluation mechanism for logic programs. The input to a typical partial deducer is a program and a partially instantiated query. The instantiated part represents the information with respect to which one would like to specialise; the uninstantiated part represents the information not yet known. Therefore, all classical partial deduction techniques use top down evaluation (or SLD-resolution) to evaluate the program parts that depend on the known input and generate a new program that computes its result using only the remainder of the input. Since the new program has less computations to perform, in general, it will be more efficient.

In recent work [21], we argued the need for a complementary partial deduction technique, capable of "specialising" a program w.r.t. a set of (unit) clauses

\footnotetext{
* Supported by a specialisation grant of the Flemish Institute for the Promotion of Scientific-Technological Research in Industry (IWT), Belgium.

** Senior Research Associate of the Belgian National Fund for Scientific Research.

$\star \star \star$ Partially supported by Esprit project 25503, ARGo.
} 
instead of a goal. It seems natural to define such a specialisation scheme in terms of bottom up evaluation. During evaluation, the information from the unit clauses is then propagated upwards, and new facts and clauses are derived. In [21], we developed a specific, and very concrete control scheme for such a bottom up transformation and provided some examples showing that a combination of bottom up transformation and classical (goal-directed) top down partial deduction achieves at least equally good results as a top down scheme alone, while requiring a much less complicated control mechanism for either part.

In this work, we establish a solid theoretical foundation for such bottom up transformation schemes, rendering a general framework in which concrete control strategies can be proven correct.

In Section 2, we first recapitulate and illustrate briefly the main motivation for bottom up specialisation. Subsequently, Section 3 - the main one - develops the framework. Next, we address concrete control in Section 4. Section 5, finally, discusses achievements and plans for future work.

Throughout the paper, we restrict attention to definite programs (and goals).

\section{Motivating Bottom Up Specialisation}

In this section, we recapitulate briefly our motivation for bottom up specialisation. More information can be found in [19,21].

In a logic program, some information flows naturally in a bottom up fashion, starting from a set of unit clauses. Take as example a program in which the concrete representation of some data structure is hidden by an abstract data type (ADT) and all operations manipulating the structure are defined using functionality provided by the ADT implementation. Abstracting such information through several layers makes sense from a software engineering point of view: concrete representations can be altered without much effort. On the other hand, every layer of abstraction decreases efficiency. Therefore, it makes sense to propagate the concrete information up into the program, to the places where it is really used. In the ADT-example: propagating the concrete representation into the program can eliminate all calls to the ADT, removing a layer of overhead.

This sort of information propagation can in principle be obtained by a top down specialisation scheme. Achieving it in a general and completely automatic way, however, is far from trivial since control information, needed by the specialiser to decide whether or not to continue the specialisation, might also flow bottom up. In particular, (Vanilla-like) meta-programs typically present diffculties of this kind [20]. Consider the following example, taken from [21]: The predicate make_list $(T, I, R)$ can be used to create a list of a fixed length (type $T$ ), with each element initialised with $I$. The result is returned in $R$.

Example 1.

fill_list $(L, T, I, L) \leftarrow \operatorname{type}(T, L)$. fill_list $(L, T, I, R) \leftarrow$ fill_list $([I \mid L], T, I, R)$.

type (list $1,[X])$

make_list $(T, I, R) \leftarrow$ fill_list $([], T, I, R)$.

$$
\text { type (list } 3,\left[X_{1}, X_{2}, X_{3}\right] \text { ). }
$$


Example 1 represents a class of recursive predicates that build up some structure between calls before a base clause of the predicate (ending the recursion) is reached, depending on the structure built. All top down specialisers are based on unfolding techniques, which are known to have problems with these: Often, it cannot be derived from the SLD-tree built so far whether or not unfolding such a recursive call will eventually terminate.

If this recursive predicate is handled in a bottom up fashion, structure is shrinking between recursive calls, resulting in the facts

make list(list $1, I,[I])$.

make list(list $3, I,[I, I, I])$.

Apart from control, there are other reasons why a bottom up approach might be preferred. Sometimes goal-directed specialisation simply is not needed, because all information to be propagated into the program is already there, and need not be provided from elsewhere. Consider a program library $M$, in which $n$ routines are defined, all using an ADT. A top down specialiser needs a single goal to start specialisation from, which might not be available when specialising the library. Or it/they will likely contain no information (all arguments free) since the latter flows bottom up (further complicating top down control).

So, in a number of cases, proceeding bottom up is a more natural solution. bottom up transformation and specialisation has been considered occasionally before (see e.g. [11,4]). However, to the best of our knowledge, our ongoing effort is the first attempt to achieve these in a completely general and automatic way.

\section{Defining the Framework}

First, we recall the concept of a non-ground $T_{P}$ operator, which normally acts on atoms. However, for reasons that will become clear below, we consider a nonground $T_{P}$ operator acting on sets of clauses ${ }^{1}$ as in compositional semantics (see e.g. $[2,1])$. To keep the presentation simple, atoms will be considered as unit clauses and vice versa, as the context requires. In what follows, $\mathcal{H C}$ denotes the set of all Horn clauses over a fixed first order language $\mathcal{L}$, underlying a given definite program $P$. Let $\equiv$ be the variant relation on $\mathcal{H C}$. For any set $S, 2^{S}$ denotes its powerset. Unless specified otherwise, uppercase characters $A, B \ldots$ denote atoms, $\tilde{A}, \tilde{B}$ conjunctions of atoms, $r, s, t, u$ terms and $\tilde{r}, \tilde{s}, \tilde{t}, \tilde{u}$ appropriate numbers of terms used as predicate arguments. As usual, $A \prec B$ denotes that $A$ is more general than $B$. We mean by $\theta=m g u\left(\left(B_{1}, \ldots, B_{m}\right),\left(C_{1}, \ldots, C_{m}\right)\right)$ that $\left(B_{1}, \ldots, B_{m}\right)$ and $\left(C_{1}, \ldots, C_{m}\right)$ are unifiable and $\theta$ is an $m g u$ of the m-tuples, so, $B_{i} \theta=C_{i} \theta$ for $i=1 \ldots m$. For a program $P$, Pred $(P)$ denotes the set of predicate names occurring in $P$. For an atom $A, \operatorname{Var}(A)$ denotes the set of variables of $A$.

First, we define a basic operator, adapted from [2].

\footnotetext{
${ }^{1}$ Hence the notation: $T_{P}^{C}$.
} 
Definition 1. The compositional, non-ground $T_{P}$-operator, $T_{P}^{C}$, is a function $T_{P}^{C}: 2^{\mathcal{H C}} / \equiv \rightarrow 2^{\mathcal{H C}} / \equiv$, defined for any $S \subset \mathcal{H C} / \equiv$ as: $T_{P}^{C}(S)=S \cup\{H \theta \leftarrow$ $\left(\tilde{L}_{1}, \ldots, \tilde{L_{n}}\right) \theta$ where $H \leftarrow B_{1}, \ldots, B_{n} \in P(n \geq 0), A_{1} \leftarrow \tilde{L}_{1}, \ldots, A_{n} \leftarrow \tilde{L}_{n}$ fresh variants of clauses in $S$ and $\theta=m g u\left(\left(B_{1}, \ldots, B_{n}\right),\left(A_{1}, \ldots, A_{n}\right)\right)$.\}

From [1], we know that $T_{P}^{C}$ is continuous and its least fixpoint $T_{P}^{C} \uparrow \omega$ exists. By definition, $T_{P}^{C} \uparrow \omega$ is a set of unit clauses denoting the least $S$-Herbrand model $[8,1]$ of $P, M_{s}(P)$. That is, $M_{s}(P)=\operatorname{Heads}\left(T_{P}^{C} \uparrow \omega\right)$ where Heads is a function that projects a set of (unit) clauses onto their heads. In the remainder of this paper, we simply consider $M_{s}(P)=T_{P}^{C} \uparrow \omega$.

Example 2. Consider the following program:

$$
\begin{array}{rlrl}
P: & p(X, c) \leftarrow & r(a) . \\
p(a, a) \leftarrow & r(f(X)) \leftarrow r(X) . \\
q(X, Y) \leftarrow p(X, Y) & & \\
\text { Then } M_{s}(P)=\{p(X, c), p(a, a), r(a), q(X, c), q(a, a), r(f(a)), r(f(f(a))), \ldots\}
\end{array}
$$

Our aim is to define a partial deduction operator that transforms a program $P$ into a program $P^{\prime}$ in which information is propagated in a bottom up fashion. $T_{P}^{C}$ can serve as a basis for such an operator, but we need to ensure that consecutive applications of our operator always terminate within finite time. To achieve this, unlike $[2,1]$, we define and incorporate an abstraction operator on $\mathcal{H C}$.

First, we introduce the concept of a predicate renaming.

Definition 2. $A$ predicate renaming of an atom $p\left(t_{1}, \ldots, t_{n}\right)$ is $p^{\prime}\left(t_{1}, \ldots, t_{n}\right)$ where either $p^{\prime}=p$, or $p^{\prime}$ denotes a new, unique predicate name.

Thus, a predicate renaming of an atom $p(\hat{t})$ is the atom itself or $p^{\prime}(\tilde{t})$, where $p^{\prime}$ is a newly introduced predicate symbol (not used before in the same program transformation).

Definition 3. An abstraction function, Abst, is an idempotent function ${ }_{2} \mathcal{H C} / \equiv \rightarrow 2^{\mathcal{H C}} / \equiv$, such that for any $S \subseteq 2^{\mathcal{H C}} / \equiv$, if $S^{\prime}=\operatorname{Abst}(S)$, then for every clause $p(\tilde{t}) \leftarrow \tilde{B} \in S$, either $p(\tilde{t}) \leftarrow \tilde{B} \in S^{\prime}$ or there exists a clause $p(\hat{s}) \leftarrow$ $p^{\prime}(\hat{s}) \in S^{\prime}$ such that $p(\hat{s}) \preceq p(\tilde{t})$ and $p^{\prime}(\hat{s})$ is a predicate renaming of $p(\hat{s})$.

As we show below, the abstraction $p(\hat{s}) \leftarrow p^{\prime}(\hat{s})$ can generate, in combination with an appropriate renaming of the original clause, $p^{\prime}(\tilde{t}) \leftarrow \tilde{B}$, the same atoms of $M_{s}(P)$ as the original clause $p(\tilde{t}) \leftarrow \tilde{B}$ (since $\theta=m g u\left(p^{\prime}(\tilde{t}), p^{\prime}(\tilde{s})\right)$ and $p(\tilde{s}) \theta=$ $p(\tilde{t}))$. Since possibly more clauses can be mapped on the same generalisation, abstraction enables us to replace the computation of $M_{s}(P)$ (which is possibly infinite) with the computation of a finite set of clauses, defining the same least S-Herbrand model.

Therefore, we combine $T_{P}^{C}$ with an abstraction function to obtain a bottom up partial deduction operator.

Definition 4. The abstracting $T_{P}^{C}$-operator, $A_{P}^{C}$, associated to a program $P$ and an abstraction function Abst is defined as $A_{P}^{C}=$ Abst $\circ T_{P}^{C}$. 
Example 3. Reconsider the program of Example 2. We define Abst $(\{p(a, a) \leftarrow\})$ $=\{p(a, Y) \leftarrow p(a, Y)\}$ and $A b s t(\{r(a)\} \cup\{r(f(X)) \leftarrow r(X)\})=\{r(X) \leftarrow r(X)\}$ while all other clauses remain unchanged by Abst. Then

$I_{0}=\{\}$

$I_{1}=A_{P}^{C}\left(I_{0}\right)=\left\{\begin{array}{l}p(a, Y) \leftarrow p(a, Y) \\ p(X, c) \leftarrow \\ r(X) \leftarrow r(X)\end{array}\right\} \quad I_{2}=A_{P}^{C}\left(I_{1}\right)=\left\{\begin{array}{l}p(a, Y) \leftarrow p(a, Y) \\ p(X, c) \leftarrow \\ r(X) \leftarrow r(X) \\ q(a, Y) \leftarrow p(a, Y) \\ q(X, c) \leftarrow\end{array}\right\}$

The reader can easily verify that $I_{2}$ is the least fixpoint of $A_{P}^{C}$.

In general, $A_{P}^{C}$ is not a monotonic operator, since $A b s t$ may replace clauses by their generalisations. However, the following proposition holds:

Proposition 1. For any $S \subseteq \mathcal{H C} / \equiv$, such that $A b s t(S)=S$, we have $S \subseteq$ $A_{P}^{C}(S)$.

Proposition 1 follows straightforwardly from the definition of $A_{P}^{C}$ and monotonicity of $T_{P}^{C}$. Thus, if we start off from an initial set $S_{0}$ which satisfies $A b s t\left(S_{0}\right)=$ $S_{0}$, monotonicity of a sequence of $A_{P}^{C}$-applications is ensured since $A b s t$ is required to be idempotent. Therefore, we can define ordinal powers of $A_{P}^{C}$ as follows:

Definition 5. $A_{P}^{C} \uparrow 0=\{\}, A_{P}^{C} \uparrow n=A_{P}^{C}\left(A_{P}^{C} \uparrow(n-1)\right)$ for $n \in \mathbb{N}$ and $A_{P}^{C} \uparrow \omega=\bigcup_{n \in \mathbb{N}} A_{P}^{C} \uparrow n$.

Henceforth, we will always use $A_{P}^{C}$ as in Definition 5. As long as no abstraction occurs, subsequent $A_{P}^{C}$ applications, starting from an initial set \{\} derive sets of unit clauses. Abstraction introduces clauses in a set $A_{P}^{C} \uparrow i$ that are no longer unit clauses, but clauses capable of generating (among others) the same atoms as the abstracted ones, provided the originals are present. To that extent, we define two complementary functions on sets generated by $A_{P}^{C}$ : $\mathcal{R}$ es, containing the clauses $p^{\prime}(\tilde{t}) \leftarrow \tilde{B}$ where $p(\tilde{t}) \leftarrow \tilde{B}$ was removed because of an abstraction $p(\hat{s}) \leftarrow p^{\prime}(\hat{s})$ and $\mathcal{G}$ en, comprising the abstractions themselves.

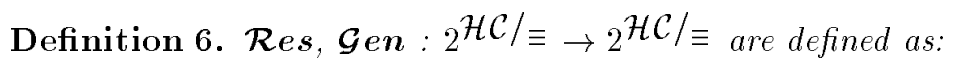

$-\boldsymbol{R e s}\left(A_{P}^{C} \uparrow 0\right)=\mathcal{G e n}\left(A_{P}^{C} \uparrow 0\right)=\{\}$.

$-\boldsymbol{\mathcal { R e s }}\left(A_{P}^{C} \uparrow n\right)=\boldsymbol{\mathcal { R e s }}\left(A_{P}^{C} \uparrow(n-1)\right) \cup\left\{p^{\prime}(\tilde{t}) \leftarrow \tilde{B} \mid p(\hat{t}) \leftarrow \tilde{B} \in T_{P}^{C}\left(A_{P}^{C} \uparrow(n-1)\right)\right.$ and $p(\tilde{t}) \leftarrow \tilde{B} \notin A_{P}^{C} \uparrow n$ and $\left.A b s t(\{p(\tilde{t}) \leftarrow \tilde{B}\})=\left\{p(\hat{s}) \leftarrow p^{\prime}(\hat{s})\right\}\right\}$ for any $n>0$.

$-\boldsymbol{G e n}\left(A_{P}^{C} \uparrow n\right)=\mathcal{G e n}\left(A_{P}^{C} \uparrow(n-1)\right) \cup A b s t\left(T_{P}^{C}\left(A_{P}^{C} \uparrow(n-1)\right)\right) \backslash T_{P}^{C}\left(A_{P}^{C} \uparrow(n-1)\right)$ for any $n>0$.

Obviously, all clauses in any $\mathcal{G}$ en set are of the form $p(\hat{s}) \leftarrow p^{\prime}(\hat{s})$. Note also that $\mathcal{G e n}\left(A_{P}^{C} \uparrow n\right) \subseteq A_{P}^{C} \uparrow n$ while $\mathcal{R e s}\left(A_{P}^{C} \uparrow n\right) \cap A_{P}^{C} \uparrow n=\{\}$. 
Example 4. In Example 3, $M_{s}\left(I_{2}\right)=\{p(X, c), q(X, c), p(a, c), q(a, c)\} \neq M_{s}(P)$. We will return to the presence of $p(a, c)$ and $q(a, c)$ in Example 5 below. Here we first note that a.o. $p(a, a)$ and $q(a, a)$ have disappeared. This is due to the abstraction of $p(a, a) \leftarrow$ into $p(a, Y) \leftarrow p(a, Y)$. There is however a clause $q(a, Y) \leftarrow p(a, Y)$ in $A_{P}^{C} \uparrow 2$ such that $\theta=m g u(p(a, a), p(a, Y))$ exists and $q(a, Y) \theta=q(a, a)$. Hence, while $p(a, a)$ is abstracted away and also $q(a, a)$ therefore not directly derived by $A_{P}^{C}$, the situation is rectified by storing $p(a, a)$ through $\mathcal{R}$ es and adding it to the final program, as in Definition 7 below.

In what follows, we will assume $A b s t$ to be defined such that $A_{P}^{C}$ is finitary ${ }^{2}$; in other words, $A_{P}^{C} \uparrow \omega=A_{P}^{C} \uparrow n_{0}$, for some $n_{0} \in \mathbb{N}$. Note that if $A_{P}^{C}$ is indeed finitary and reaches its least fixpoint in $n_{0} \in \mathbb{N}, \mathcal{R e s}\left(A_{P}^{C} \uparrow n_{0}\right)$ and $\mathcal{G e n}\left(A_{P}^{C} \uparrow n_{0}\right)$ are necessarily finite sets, since all sets $A_{P}^{C} \uparrow n, n \leq n_{0}$ are finite.

We are now in a position to define the notion of a bottom up partial deduction.

Definition 7. Given a program $P$ and an abstraction function Abst, giving rise to a finitary abstracting $T_{P}^{C}$-operator, $A_{P}^{C}$, with least fixpoint $A_{P}^{C} \uparrow n_{0}$, the bottom up partial deduction of $P$ (using $A b s t$ ) is the program

$A_{P}^{C} \uparrow n_{0} \cup \mathcal{R} \operatorname{es}\left(A_{P}^{C} \uparrow n_{0}\right)$.

Before reconsidering our example, we return to the use of predicate-renamings in the abstraction function. Notice that by Definition 2, a valid predicate renaming of an atom $A$ is the atom itself. As a consequence, clauses in $\mathcal{G}$ en (and hence in the residual program, $\left.P^{\prime}\right)$ may be of the form $p(\hat{s}) \leftarrow p(\hat{s})$. Such clauses are "useless" and possibly introduce non-termination in top down evaluation. However, since our transformation is based on the $T_{P}^{C}$-operator, it seems natural to formulate soundness and completeness of bottom up partial deduction in terms of least S-Herbrand models, and the presence of such clauses in $P^{\prime}$ does not influence $M_{s}\left(P^{\prime}\right)$. Moreover, after completion of the transformation, they can be easily excluded from the residual program. However, as Example 5 below shows, renamings are sometimes necessary to guarantee soundness of the transformation. First, we recapitulate the definition of soundness and completeness with respect to the least S-Herbrand model. Obviously, also in the transformed program, we are really only interested in the original predicates. Hence, in the remainder of this paper and in the following definition of transformation correctness, $M_{s}\left(P^{\prime}\right)_{\mid \operatorname{Pred}(P)}=\left\{p(\tilde{t}) \in M_{s}\left(P^{\prime}\right) \mid p \in \operatorname{Pred}(P)\right\}$.

Definition 8. Let $P^{\prime}$ be a bottom up partial deduction of a program $P . P^{\prime}$ is sound w.r.t. $P$ if $M_{s}\left(P^{\prime}\right)_{\mid \operatorname{Pred}(P)} \subseteq M_{s}(P) . P^{\prime}$ is complete w.r.t. $P$ if $M_{s}(P) \subseteq$ $M_{s}\left(P^{\prime}\right)$.

Without the use of predicate renamings, a bottom up partial deduction is not sound in general.

\footnotetext{
${ }^{2}$ Clearly, this is a matter of control: It involves deciding when to abstract and how to
} do it. See Section 4. 
Example 5. Reconsider the program $P$ from Example 2 and Abst from Example 3. Since $A_{P}^{C}\left(I_{2}\right)=I_{2}$, the fixpoint is reached in $A_{P}^{C} \uparrow 2$ and the bottom up partial deduction of $P$ is $P^{\prime}=I_{2} \cup \mathcal{R} \operatorname{es}\left(I_{2}\right)$ and $\mathcal{R e s}\left(I_{2}\right)=\{p(a, a) \leftarrow\} \cup\{r(a) \leftarrow\}$ $\cup\{r(f(X)) \leftarrow r(X)\}$. It is easy to see that, while $P$ and $P^{\prime}$ have equal least Herbrand models, $M_{s}\left(P^{\prime}\right) \not \subset M_{s}(P)$ :

$$
\begin{gathered}
M_{s}(P)=\{p(X, c), p(a, a), r(a), q(X, c), q(a, a), r(f(a)), r(f(f(a))), \ldots\} \\
M_{s}\left(P^{\prime}\right)=\{p(X, c), p(a, a), r(a), q(X, c), q(a, a), \boldsymbol{p}(\boldsymbol{a}, \boldsymbol{c}), q(\boldsymbol{a}, c), r(f(a)), \ldots\} .
\end{gathered}
$$

In this example, due to the abstraction of $p(a, a) \leftarrow$ into $p(a, Y) \leftarrow p(a, Y)$, we introduce a clause in $P^{\prime}$ that is capable of further instantiating elements of $M_{s}\left(P^{\prime}\right): p(X, c) \in M_{s}\left(P^{\prime}\right)$ and $p(X, c)$ unifies with $p(a, Y)$ without being an instance of it, and hence $p(a, c) \in M_{s}\left(P^{\prime}\right)$ while it is not an element of $M_{s}(P)$. Soundness can be obtained by imposing an extra condition on a bottom up partial deduction, ensuring that if any element $p^{\prime}(\tilde{t})$ of $M_{s}\left(P^{\prime}\right)$ unifies with the body atom of a clause $p(\tilde{s}) \leftarrow p^{\prime}(\tilde{s})$ introduced by $A b s t, p^{\prime}(\tilde{t})$ must be an instance of $p^{\prime}(\hat{s})$.

Definition 9. Let $S$ be a set of clauses, such that $\mathcal{G e n}(S)$ and $\mathcal{R e s}(S)$ are defined. $S$ is inside-closed if for all $A \leftarrow \tilde{B} \in S \cup \mathcal{R e s}(S)$ holds: if $H \leftarrow H^{\prime} \in$ $\mathcal{G e n}(S)$ such that $\theta=\operatorname{mgu}\left(A, H^{\prime}\right)$ exists, then $H^{\prime} \theta=A$.

As shown in Example 5, when this condition is not imposed on $A_{P}^{C} \uparrow n_{0}$, Abst might create clauses by which atoms in $M_{s}\left(P^{\prime}\right)$ are derived that are more instantiated than any atom in $M_{s}(P)$. Note that a concrete control strategy (as in Section 4, e.g.) need not actually rename as long as the set $A_{P}^{C} \uparrow i$ stays inside-closed. Hence the possibility to re-use the same predicate in Definition 2.

Example 6. Reconsider the program $P$ from Example 2 but Abst now defined as $A b s t(\{p(a, a) \leftarrow\})=\left\{p(a, Y) \leftarrow p^{\prime}(a, Y)\right\}$ and $A b s t(\{r(a) \leftarrow\} \cup\{r(f(X)) \leftarrow$ $r(X)\})=\{r(X) \leftarrow r(X)\}$ (Abst introduces the new predicate $\left.p^{\prime}\right)$. The new transformed program is $P^{\prime}=A_{P}^{C} \uparrow 2 \cup \mathcal{R} \operatorname{es}\left(A_{P}^{C} \uparrow 2\right)$ and $\mathcal{R e s}\left(A_{P}^{C} \uparrow 2\right)=$ $\left\{p^{\prime}(a, a) \leftarrow\right\} \cup\{r(a) \leftarrow\} \cup\{r(f(X)) \leftarrow r(X)\}$ where $P^{\prime}$ contains now $p(a, Y) \leftarrow$ $p^{\prime}(a, Y)$. Hence,

$$
M_{s}\left(P^{\prime}\right)_{\mid \operatorname{Pred}(P)}=M_{s}(P)=\{p(X, c), p(a, a), r(a), q(X, c), q(a, a), r(f(a)), \ldots\} .
$$

Now, we can prove the correctness of the transformation.

Theorem 1. Let $P^{\prime}$ be a bottom up partial deduction of a program $P, P^{\prime}=$ $A_{P}^{C} \uparrow n_{0} \cup \mathcal{R e s}\left(A_{P}^{C} \uparrow n_{0}\right)$. Then, if $A_{P}^{C} \uparrow n_{0}$ is inside-closed, $M_{s}\left(P^{\prime}\right)_{\mid \operatorname{Pred}(P)}=$ $M_{s}(P)$, thus $P^{\prime}$ is sound and complete w.r.t. $P$.

In order to prove this theorem, we include a useful lemma from [6].

Lemma 1. (Proposition II.1 from [6])

Let $\mathcal{A}=\left\{A_{i}\right\}_{1 \leq i \leq n}$ be a set of positive literals, $\mathcal{B}=\left\{B_{i} \leftarrow B_{i, 1}, \ldots, B_{i, n_{i}}\right\}_{1 \leq i \leq n}$ and $\mathcal{C}=\left\{C_{i, j} \leftarrow C_{i, j, 1}, \ldots, C_{i, j, p_{i, j}}\right\}_{1 \leq i \leq n, 1 \leq j \leq n_{i}}$ be sets of definite clauses. Let 
us further assume that $\operatorname{Var}(\mathcal{A}), \operatorname{Var}(\mathcal{B})$ and $\operatorname{Var}(\mathcal{C})$ are disjoint sets. Then, there exist substitutions $\alpha$ and $\beta$ such that:

$$
(I)\left\{\begin{array}{l}
\alpha=\operatorname{mgu}\left(\left(A_{i}\right)_{1 \leq i \leq n},\left(B_{i}\right)_{1 \leq i \leq n}\right), \\
\beta=\operatorname{mgu}\left(\left(B_{i, j} \alpha\right)_{1 \leq i \leq n, 1 \leq j \leq n_{i}},\left(C_{i, j}\right)_{1 \leq i \leq n, 1 \leq j \leq n_{i}}\right)
\end{array}\right.
$$

if and only if there exists substitutions $\gamma$ and $\delta$ such that:

$$
(I I)\left\{\begin{array}{l}
\gamma=m g u\left(\left(B_{i, j}\right)_{1 \leq i \leq n, 1 \leq j \leq n_{i}},\left(C_{i, j}\right)_{1 \leq i \leq n, 1 \leq j \leq n_{i}}\right), \\
\delta=m g u\left(\left(A_{i}\right)_{1 \leq i \leq n},\left(B_{i} \gamma\right)_{1 \leq i \leq n}\right)
\end{array}\right.
$$

Moreover, if $(I)$ or (II) holds then $\alpha \beta=\gamma \delta$ (modulo variable renaming).

The following corollary states that if $m g u\left(\left(A_{i}\right)_{1 \leq i \leq n},\left(B_{i}\right)_{1 \leq i \leq n}\right)$ exists, so does $m g u\left((H)_{1 \leq i \leq n},\left(B_{i}\right)_{1 \leq i \leq n}\right)$ if $H_{i} \preceq A_{i}(1 \leq i \leq n)$ and $\operatorname{Var}\left(\left(A_{i}\right)_{1 \leq i \leq n}\right)$, $\operatorname{Var}\left(\left(B_{i}\right)_{1 \leq i \leq n}\right) \overline{)}$ and $\operatorname{Var}\left((\bar{H})_{1 \leq i \leq n}\right)$ are disjoint sets.

Corollary 1. Let $\left(A_{1}, \ldots, A_{n}\right),\left(B_{1}, \ldots, B_{n}\right)$ and $\left(H_{1}, \ldots, H_{n}\right)$ be sets of atoms with $\operatorname{Var}\left(A_{1}, \ldots, A_{n}\right), \operatorname{Var}\left(B_{1}, \ldots, B_{n}\right)$ and $\operatorname{Var}\left(H_{1}, \ldots, H_{n}\right)$ disjoint sets such that $\left(A_{1}, \ldots, A_{n}\right)=\left(H_{1}, \ldots, H_{n}\right) \gamma$. Then, if $m g u\left(\left(A_{1}, \ldots, A_{n}\right),\left(B_{1}, \ldots, B_{n}\right)\right)$ exists, so does $m g u\left(\left(H_{1}, \ldots, H_{n}\right),\left(B_{1}, \ldots, B_{n}\right)\right)$.

Proof. Apply the only-if of Lemma 1 with $\mathcal{A}=\left\{A_{1}, \ldots, A_{n}\right\}, \mathcal{C}=\left\{B_{1}, \ldots, B_{n}\right\}$ (seen as unit clauses) and $\mathcal{B}$ the set of clauses $\left\{H_{1} \leftarrow H_{1}, \ldots, H_{n} \leftarrow H_{n}\right\}$.

Below, in order to avoid uninteresting technical details, we assume renaming apart and reason modulo variable renaming wherever appropriate.

\section{Proof of Theorem 1}

Proof. Soundness. Since every step in the computation of $A_{P}^{C}$ represents a Horn clause program, we introduce the following definition of intermediate programs: $P_{0}=\{\}$, $P_{i}=A_{P}^{C} \uparrow i \cup \mathcal{R} \operatorname{es}\left(A_{P}^{C} \uparrow i\right)$. We assume that $P^{\prime}=A_{P}^{C} \uparrow n \cup \mathcal{R} \operatorname{es}\left(A_{P}^{C} \uparrow n\right)$ and prove by induction that for all $i \leq n: M_{s}\left(P_{i}\right)_{\mid \operatorname{Pred}(P)} \subseteq M_{s}(P)$. Obviously, $M_{s}\left(P_{0}\right) \subseteq M_{s}(P)$. Suppose now that $M_{s}\left(\bar{P}_{k}\right)_{\mid \operatorname{Pred}(P)} \subseteq M_{s}(P)$ (Induction Hypothesis). The difference between $P_{k+1}$ and $P_{k}$ consists of:

1. clauses added by $T_{P}^{C}$ : Consider $H_{1} \leftarrow \tilde{L}_{1}, \ldots, H_{n} \leftarrow \tilde{L}_{n}$ in $A_{P}^{C} \uparrow k$ and a clause $H \leftarrow$ $B_{1}, \ldots, B_{n}$ in $P$ such that $\alpha=m g u\left(\left(B_{1}, \ldots, B_{n}\right),\left(H_{1}, \ldots, H_{n}\right)\right)$ exists and $H \alpha \leftarrow$ $\left(\tilde{L}_{1}, \ldots, \tilde{L}_{n}\right) \alpha \in A_{P}^{C} \uparrow(k+1)$. We have to prove that for each conjunction of atoms, $\tilde{D}$ in $M_{s}\left(P^{\prime}\right)$ holds that if $\beta=\operatorname{mgu}\left(\tilde{D},\left(\tilde{L}_{1}, \ldots, \tilde{L}_{n}\right) \alpha\right), H \alpha \beta \in M_{s}(P)$. By Lemma 1 , the existence of $\alpha$ and $\beta$ implies the existence of $\gamma=m g u\left(\left(\tilde{L}_{1}, \ldots, \tilde{L}_{n}\right), \tilde{D}\right)$ and $\delta=m g u\left(\left(H_{1}, \ldots, H_{n}\right) \gamma,\left(B_{1}, \ldots, B_{n}\right)\right)$. By induction hypothesis, $H_{1} \gamma, \ldots, H_{n} \gamma \in$ $M_{s}(P)$, and since $H \leftarrow B_{1}, \ldots, B_{n}$ is a clause of $P, H \delta=H \gamma \delta=H \alpha \beta \in M_{s}(P)$.

2. clauses $p(\tilde{t}) \leftarrow \tilde{B}$ removed by $A b s t$ and recovered through $\mathcal{R} e s$ as $p^{\prime}(\tilde{t}) \leftarrow \tilde{B}$ with $p^{\prime}(\hat{t})$ a predicate renaming of $p(\tilde{t})$. Obviously, for each conjunction of atoms, $\tilde{D}$ in $M_{s}\left(P^{\prime}\right)$ such that $\beta=\operatorname{mgu}(\tilde{D}, \tilde{B}), p^{\prime}(\tilde{s}) \beta \in M_{s}\left(P^{\prime}\right)$ and $p(\tilde{s}) \beta \in M_{s}(P)$ (since the induction hypothesis holds for the original clause).

3. clauses $p(\hat{s}) \leftarrow p^{\prime}(\hat{s})$ introduced by $A b s t$ (with $p^{\prime}(\hat{s})$ a predicate renaming of $p(\hat{s}))$ : By (2), we know that if $p^{\prime}(\hat{t}) \in M_{s}\left(P^{\prime}\right), p(\hat{t}) \in M_{s}(P)$. Since abstraction must guarantee inside-closedness, if $\theta=m g u\left(p^{\prime}(\hat{s}), p^{\prime}(\tilde{t})\right), p^{\prime}(\hat{s}) \theta=p^{\prime}(\tilde{t})$ and thus $p(\tilde{s}) \theta=p(\tilde{t}) \in M_{s}(P)$. 
Completeness. We prove by induction that for every $k, T_{P}^{C} \uparrow k \subseteq M_{s}\left(P^{\prime}\right)$. Since

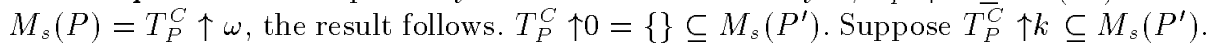
By monotonicity of $T_{P}^{C}$ we need to consider only clauses added in the $(k+1)$-th application of $T_{P}^{C}: T_{P}^{C} \uparrow(k+1) \backslash T_{P}^{C} \uparrow k=\left\{H \delta \leftarrow\right.$ true $\mid H \leftarrow B_{1}, \ldots, B_{n} \in P, A_{1} \leftarrow$ true $, \ldots, A_{n} \leftarrow$ true $\in T_{P}^{C} \uparrow k$, and $\left.\delta=\operatorname{mgu}\left(\left(B_{1}, \ldots, B_{n}\right),\left(A_{1}, \ldots, A_{n}\right)\right)\right\}$. We need to prove that $H \delta \in M_{s}\left(P^{\prime}\right)$. Since $A_{1}, \ldots, A_{n} \in T_{P}^{C} \uparrow k, A_{1}, \ldots, A_{n} \in M_{s}\left(P^{\prime}\right)$ (by induction hypothesis). This implies the existence of clauses $H_{1} \leftarrow \tilde{L}_{1}, \ldots, H_{n} \leftarrow \tilde{L}_{n}$ in $P^{\prime}$ and conjunctions of atoms $\tilde{D}_{1}, \ldots, \tilde{D}_{n} \in M_{s}\left(P^{\prime}\right)$ such that

$$
\gamma=\operatorname{mgu}\left(\left(\tilde{D}_{1}, \ldots, \tilde{D}_{n}\right),\left(\tilde{L}_{1}, \ldots, \tilde{L}_{n}\right)\right)
$$

and $\left(H_{1}, \ldots, H_{n}\right) \gamma=\left(A_{1}, \ldots, A_{n}\right)$. Since $H_{1} \leftarrow \tilde{L}_{1}, \ldots, H_{n} \leftarrow \tilde{L}_{n}$ are clauses of $P^{\prime}$, there exists an $m$ such that some of these clauses are in $A_{P}^{C} \uparrow m$, and the others in $\mathcal{R} \operatorname{es}\left(A_{P}^{C} \uparrow m\right)$. Let us assume that (possibly after renumbering) $H_{1} \leftarrow \tilde{L}_{1}, \ldots, H_{k} \leftarrow \tilde{L}_{k} \in$ $A_{P}^{C} \uparrow m$ and $H_{k+1} \leftarrow \tilde{L}_{k+1}, \ldots, H_{n} \leftarrow \tilde{L}_{n} \in \mathcal{R} \operatorname{es}\left(A_{P}^{C} \uparrow m\right)$. Since the latter clauses have been abstracted in the process of computing $A_{P}^{C} \uparrow m$, there exist clauses $H_{k+1}^{\prime} \leftarrow$ $H_{k+1}^{\prime}, \ldots, H_{n}^{\prime} \leftarrow H_{n}^{\prime}$ in $A_{P}^{C} \uparrow m$ such that

$$
\left(H_{k+1}^{\prime}, \ldots, H_{n}^{\prime}\right) \eta=H_{k+1}, \ldots, H_{n} .
$$

Since

$$
\delta=m g u\left(\left(B_{1}, \ldots, B_{n}\right),\left(A_{1}, \ldots, A_{n}\right)\right)
$$

and $\left(A_{1}, \ldots, A_{n}\right)=\left(H_{1}, \ldots, H_{n}\right) \gamma, m g u\left(\left(B_{1}, \ldots, B_{n}\right),\left(H_{1}, \ldots, H_{n}\right)\right)$ exists (Corollary 1). Next, again applying Corollary 1 , since

$$
\begin{gathered}
\left(H_{1}, \ldots, H_{n}\right)=\left(H_{1}, \ldots, H_{k}, H_{k+1}^{\prime}, \ldots, H_{n}^{\prime}\right) \eta, \\
\theta=\operatorname{mgu}\left(\left(B_{1}, \ldots, B_{n}\right),\left(H_{1}, \ldots, H_{k}, H_{k+1}^{\prime}, \ldots, H_{n}^{\prime}\right)\right)
\end{gathered}
$$

exists. Recall that

$$
H \leftarrow B_{1}, \ldots, B_{n} \in P \text { and }\left\{\begin{array}{l}
H_{1} \leftarrow \tilde{L}_{1} \\
\cdots \\
H_{k} \leftarrow \tilde{L}_{k} \\
H_{k+1}^{\prime} \leftarrow H_{k+1}^{\prime} \\
\cdots \\
H_{n}^{\prime} \leftarrow H_{n}^{\prime}
\end{array} \in A_{P}^{C} \uparrow m\right.
$$

Together (2) and (3) imply that $H \theta \leftarrow\left(\tilde{L}_{1}, \ldots, \tilde{L}_{k}, H_{k+1}^{\prime}, \ldots, H_{n}^{\prime}\right) \theta \in T_{P}^{C}\left(A_{P}^{C} \uparrow m\right)$ and hence is a clause of $P^{\prime}$. Now, from $\left(H_{k+1}^{\prime}, \ldots, H_{n}^{\prime}\right) \eta=\left(H_{k+1}, \ldots, H_{n}\right)$ it follows that there is an $\eta^{\prime}=m g u\left(\left(H_{k+1}^{\prime}, \ldots, H_{n}^{\prime}\right),\left(H_{k+1} \gamma, \ldots, H_{n} \gamma\right)\right)$ where $\gamma$ is defined as in (1). Moreover, (1) implies the existence of

$$
\gamma^{\prime}=\operatorname{mgu}\left(\left(\tilde{L}_{1}, \ldots, \tilde{L}_{k}\right),\left(\tilde{D}_{1}, \ldots, \tilde{D}_{k}\right)\right) .
$$

Since $\operatorname{Var}\left(H_{k+1}^{\prime}\right), \ldots, \operatorname{Var}\left(H_{n}^{\prime}\right), \operatorname{Var}\left(H_{k+1} \gamma\right), \ldots, \operatorname{Var}\left(H_{n} \gamma\right), \operatorname{Var}\left(\tilde{L}_{1}\right), \ldots$,

$\operatorname{Var}\left(\tilde{L}_{k}\right), \operatorname{Var}\left(\tilde{D}_{1}\right), \ldots, \operatorname{Var}\left(\tilde{D}_{k}\right)$ are disjoint sets, $\eta^{\prime}$ and $\gamma^{\prime}$ can be composed into $\eta^{\prime} \gamma^{\prime}$ :

$$
\eta^{\prime} \gamma^{\prime}=\operatorname{mgu}\left(\left(\tilde{L}_{1}, \ldots, \tilde{L}_{k}, H_{k+1}^{\prime}, \ldots, H_{n}^{\prime}\right),\left(\tilde{D}_{1}, \ldots, \tilde{D}_{k}, H_{k+1} \gamma, \ldots, H_{n} \gamma\right)\right) .
$$

Since

$$
\begin{aligned}
& \left(H_{1}, \ldots, H_{k}, H_{k+1}^{\prime}, \ldots, H^{\prime} n\right) \eta^{\prime} \gamma^{\prime} \\
& =\left(H_{1} \gamma^{\prime}, \ldots, H_{k} \gamma^{\prime}, H_{k+1} \gamma \gamma^{\prime}, \ldots, H_{n} \gamma \gamma^{\prime}\right) \\
& =\left(H_{1} \gamma, \ldots, H_{k} \gamma, H_{k+1} \gamma, \ldots, H_{n} \gamma\right) \\
& =A_{1}, \ldots, A_{n}
\end{aligned}
$$


and $\delta=m g u\left(\left(B_{1}, \ldots, B_{n}\right),\left(A_{1}, \ldots, A_{n}\right)\right)$,

$$
\delta=m g u\left(\left(B_{1}, \ldots, B_{n}\right),\left(H_{1}, \ldots, H_{k}, H_{k+1}^{\prime}, \ldots, H_{n}^{\prime}\right) \eta^{\prime} \gamma^{\prime}\right)
$$

Using the if-part of Lemma 1 with $\mathcal{A}=\left\{B_{1}, \ldots, B_{n}\right\}, \mathcal{B}$ the $A_{P}^{C} \uparrow m$-clauses of (3) and as $\mathcal{C}$ the set $\left\{\tilde{D}_{1}, \ldots, \tilde{D}_{k}, H_{k+1} \gamma, \ldots, H_{n} \gamma\right\}$ (seen as a set of unit clauses), the existence of $\eta^{\prime} \gamma^{\prime}$ and $\delta$ implies the existence of $\theta$ (as in (2)) and

$$
\sigma=m g u\left(\left(\tilde{L_{1}}, \ldots, \tilde{L}_{k}, H_{k+1}^{\prime}, \ldots, H_{n}^{\prime}\right) \theta,\left(\tilde{D}_{1}, \ldots, \tilde{D}_{k}, H_{k+1} \gamma, \ldots, H_{n} \gamma\right)\right) .
$$

$\tilde{D}_{1}, \ldots, \tilde{D}_{k} \in M_{s}\left(P^{\prime}\right)$ and $H_{k+1} \gamma, \ldots, H_{n} \gamma=\left(A_{k+1}, \ldots, A_{n}\right) \in M_{s}\left(P^{\prime}\right)$ and $H \theta \leftarrow$ $\left(\tilde{L_{1}}, \ldots, \tilde{L_{k}}, H_{k+1}^{\prime}, \ldots, H_{n}^{\prime}\right) \theta$ is a clause of $P^{\prime}$. It follows that $H \theta \sigma \in M_{s}\left(P^{\prime}\right), H \theta \sigma=$ $H \eta^{\prime} \gamma^{\prime} \delta$ through Lemma 1 and $H \eta^{\prime} \gamma^{\prime} \delta=H \delta$ since $H \eta^{\prime} \gamma^{\prime}=H$. Therefore, we have shown that $H \delta \in M_{s}\left(P^{\prime}\right)$.

In $[8,1,2]$ the equivalence between the operational semantics and the $\mathrm{S}$ semantics is established. This important result guarantees that if $M_{s}(P)=$ $M_{s}\left(P^{\prime}\right)$, the operational behavior of $P$ and $P^{\prime}$ is essentially the same: For any goal $G, P \cup G$ has an SLD-refutation with c.a.s. $\theta$ if and only if $P^{\prime} \cup G$ has an SLD-refutation with c.a.s. $\theta$. Since all classical, top down, partial deduction techniques are formulated with respect to this operational semantics, this equivalence result together with Theorem 1 enables an integration of the two transformations.

Note that while our transformation preserves finite failures, it may convert infinite failure into finite failure, as the following example shows:

Example 7.

$$
\begin{aligned}
& P: \quad q(a) \leftarrow \\
& q(X) \leftarrow q(X) \\
& P^{\prime}: \quad q(a) \leftarrow
\end{aligned}
$$

The query $q(b)$ fails infinitely in the original program $P$, whereas it fails finitely in the transformed program $P^{\prime}$.

\section{Concrete Control}

In order to show how our framework can be used in practice, we now cast a concrete bottom up control strategy within it. The basic strategy was already operationally described in [21], but by presenting it within our conceptual framework, we are now able to derive an algorithm which is operationally both sound and complete (which was not completely the case in [21]).

Example 8. Consider the following set of clauses, $\mathcal{C}$, defining two well-known predicates append and reverse working with an abstract data type for list representation.

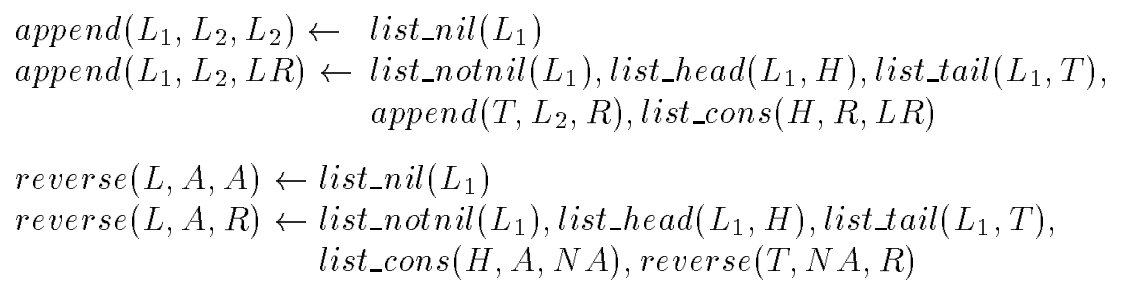


The ADT itself is represented by the following set of clauses, $\mathcal{A}$, using the classical concrete list representation.

$$
\begin{array}{ll}
\text { list_nil }([) \leftarrow & \text { list_head }\left(\left[X \mid X_{s}\right], X\right) \leftarrow \\
\text { list_notnil }\left(\left[X \mid X_{s}\right]\right) \leftarrow \quad & \text { list_tail }\left(\left[X \mid X_{s}\right], X_{s}\right) \leftarrow \\
& \text { list_cons }\left(X, X_{s},\left[X \mid X_{s}\right]\right) \leftarrow
\end{array}
$$

The program $P=\mathcal{C} \cup \mathcal{A}$ can be seen as a library that we want to specialise w.r.t. the ADT represented by $\mathcal{A}$.

In top down partial deduction, ordering partially deduced atoms in treestructures rather than sets is a commonly used control tool $[17,18,15]$. Likewise, rather than just using $T_{P}^{C}$ to derive sets of clauses, we define a new operator, $D_{P}$, which orders the derived clauses into a DAG. By doing so, we make the relation between derived clauses explicit, facilitating later on the formulation of a sufficiently precise abstraction function. We denote with $\mathcal{D}_{\mathcal{H C}}$ the set of all such DAGs, and with Clauses a function $\mathcal{D}_{\mathcal{H C}} \rightarrow 2^{\mathcal{H C}}$, mapping a DAG to the set of clauses present in it. Before defining $D_{P}$, we introduce an intermediate function, Add:

Definition 10. Add : $\mathcal{D}_{\mathcal{H C}} \rightarrow \mathcal{D}_{\mathcal{H C}}$ is a function such that for $D \in \mathcal{D}_{\mathcal{H C}}$, $\left\{C_{1}, \ldots, C_{n}\right\} \subseteq$ Clauses $(D)$ and $C \in \mathcal{H C}, A d d\left(D,\left\{C_{1}, \ldots, C_{n}\right\}, C\right)$ denotes a DAG $D^{\prime} \in \mathcal{D}_{\mathcal{H C}}$ that is the result of adding $C$ to $D$, with an edge from every $C_{i} \rightarrow C$ for $(1 \leq i \leq n)$.

Definition 11. For a definite program $P$, the $D_{P}$-operator is a function, $D_{P}$ : $\mathcal{D}_{\mathcal{H C}} \rightarrow \mathcal{D}_{\mathcal{H C}}$ defined for any DAG $D \in \mathcal{D}_{\mathcal{H C}}$ as $D_{P}(D)=D^{\prime}$, where $D^{\prime}$ is the result of repeatedly applying $A d d\left(D,\left\{C_{1}, \ldots, C_{n}\right\}, C\right)$ for every $C \in T_{P}^{C}($ Clauses $(D)) \backslash$ Clauses $(D)$ where $C$ was derived from $C_{1}, \ldots, C_{n}$ by $T_{P}^{C}$.

$D_{P}$ is continuous and monotonic, and we define its ordinal powers as usual.

Example 9. With $P$ defined as in Example 8, $D_{P} \uparrow 1, D_{P} \uparrow 2$ and $D_{P} \uparrow 3$ are depicted in Figures 1, 2 and 3 (see Appendix A) respectively.

The relation between $D_{P}$ and $T_{P}^{C}$ is obvious: For any DAG $D \in \mathcal{D}_{\mathcal{H C}}$ :

$$
\text { Clauses }\left(D_{P}(D)\right)=T_{P}^{C}(\text { Clauses }(D)) \text { holds. }
$$

This implicitly defines $\mathcal{G} \boldsymbol{e n}(D)$ and $\mathcal{R} \boldsymbol{e s}(D)$ for any $D$ constructed through $A D_{P}$, defined below. Henceforth, we will often leave implicit the distinction between the DAG and the set of clauses it contains.

We want our abstraction function to abstract clauses that would otherwise lead to an infinite DAG. To that extent, following the terminology of [15] (and references therein), we first define a well-quasi relation and give a concrete instance of such a relation that can be used as a criterion to decide when to perform abstraction. 
Definition 12. We call a relation $\mathcal{W} \subseteq \mathcal{H C} / \equiv \times \mathcal{H C} / \equiv$ well-quasi on $\mathcal{H C}$ if and only if any infinite series of clauses $C_{1}, \ldots, C_{k}, C_{k+1}, \ldots \in \mathcal{H C} / \equiv$ contains two clauses $C_{i}$ and $C_{j}, i \leq j$, such that $\left(C_{i}, C_{j}\right) \in \mathcal{W}$.

A well-known example of a well-quasi relation is the homeomorphic embedding relation. Basically, it is a well-quasi order on a finite alphabet (e.g. [7, 18]), but the refined "strict" version below is not transitive. It remains well-quasi, though [15]. The following definition is taken from $[14,15,10]$.

Definition 13. Let $X, Y$ range over variables, $f$ over functors, and $p$ over predicates. As usual, $e_{1} \prec e_{2}$ denotes that $e_{2}$ is a strict instance of $e_{1}$. Define $\unlhd$ on terms and atoms:

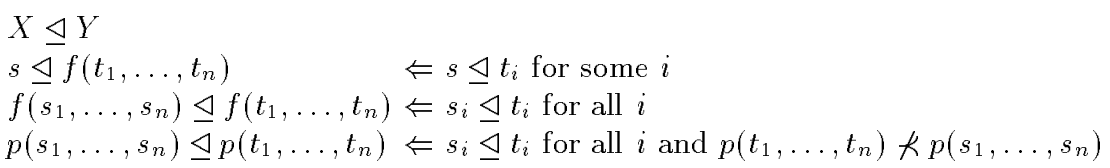

The basic intuition behind the homeomorphic embedding relation is that $A \unlhd B$ if $A$ can be obtained from $B$ (modulo variable renaming) by striking-out some functors in $B$. As such, it provides a starting point for detecting growing structure and hence possibly non-terminating processes.

Example 10. In the DAG of Example 9: reverse $([], R, R) \unlhd$ reverse $([X], A,[X \mid A])$ and append $([], L, L) \unlhd \operatorname{append}([X], L,[X \mid L])$.

Based on $\unlhd$, we can define a well-quasi relation on $\mathcal{H C}$ :

Definition 14. $\mathcal{W}_{\unlhd} \subseteq \mathcal{H C} / \equiv \times \mathcal{H C} / \equiv=\left\{\left(H_{1} \leftarrow \tilde{B}_{1}, H_{2} \leftarrow \tilde{B}_{2}\right) \mid H_{1} \unlhd H_{2}\right\}$

Given a well-quasi relation $\mathcal{W}$ on $\mathcal{H C}$, we are in a position to define an abstraction function $A_{b s t}: \mathcal{D}_{\mathcal{H C}} \rightarrow \mathcal{D}_{\mathcal{H C}}$ through the algorithm given below. It uses operations remove $(D, C): \mathcal{D}_{\mathcal{H C}} \times \mathcal{H C} \rightarrow \mathcal{D}_{\mathcal{H C}}$ which removes a clause $C$ from a DAG $D$ and replace $\left(D, C, C^{\prime}\right): \mathcal{D}_{\mathcal{H C}} \times \mathcal{H C} \times \mathcal{H C} \rightarrow \mathcal{D}_{\mathcal{H C}}$ replacing a clause $C$ in a DAG $D$ by $C^{\prime}$. Given atoms $A$ and $B, m s g(A, B)$ denotes their most specific generalisation (see a.o. $[18,15]$ ).

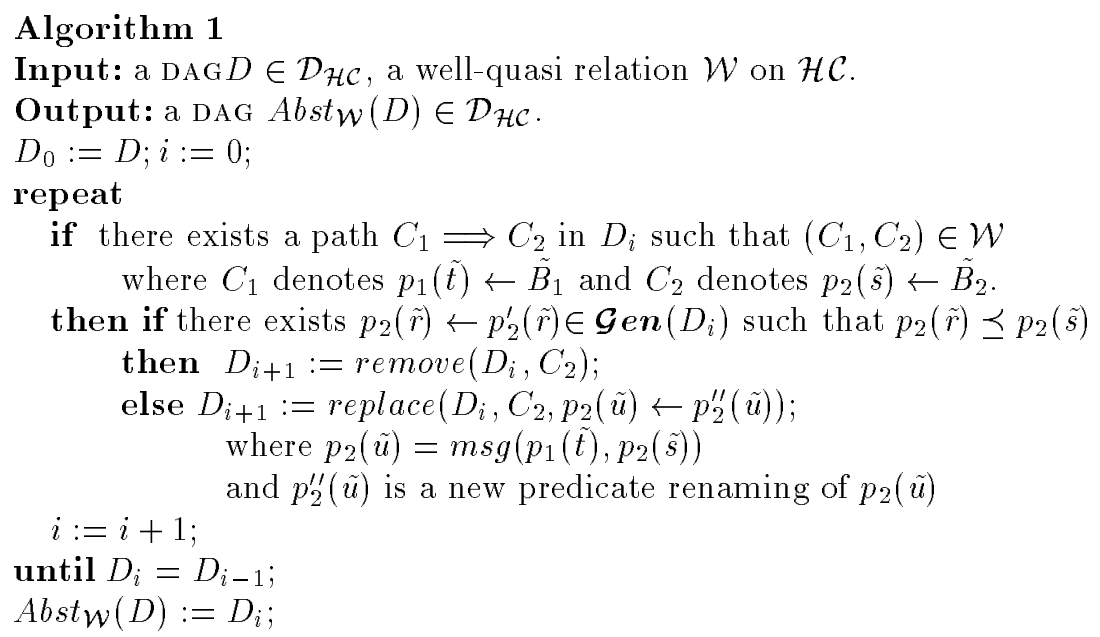


Definition 15. We call a well-quasi relation $\mathcal{W}$ on $\mathcal{H C}$ suitable (for abstraction) if $(A, B) \in \mathcal{W}$ implies msg $(A, B) \neq B$.

Note that strict homeomorphic embedding as defined in Definitions 13 and 14 is suitable [15]. Now we can state the following correctness results.

Theorem 2. Given a suitable well-quasi relation $\mathcal{W}$ on $\mathcal{H C}$, Algorithm 1 terminates for any input $\mathrm{DAG} D \in \mathcal{D}_{\mathcal{H C}}$.

Proof. In the algorithm, $D_{i} \neq D_{i-1}$ as long as there exists a path $C_{1} \Longrightarrow C_{2}$ in $D_{i}$ such that $\left(C_{1}, C_{2}\right) \in \mathcal{W}$. In each loop, either $C_{2}$ is removed from the DaG, resulting in precisely one path less to consider in the next loop, or a generalisation is made. Thus, the only thing left to prove is that there cannot occur an infinite sequence of consecutive generalisations. This follows because suitability of $\mathcal{W}$ implies that generalising is strict and therefore well-founded.

It can be easily verified that, given a suitable $\mathcal{W}, A b s t_{\mathcal{W}}$ as defined in the above algorithm complies with Definition 3 , and hence is an abstraction function, Abst $t_{\mathcal{W}}: \mathcal{D}_{\mathcal{H C}} \rightarrow \mathcal{D}_{\mathcal{H C}}$. Again, we combine $A b s t_{\mathcal{W}}$ with $D_{P}$ to obtain a concretisation of $A_{P}^{C}: A D_{P}=A b s t_{\mathcal{W}} \circ D_{P}$ and $A D_{P}: \mathcal{D}_{\mathcal{H C}} \rightarrow \mathcal{D}_{\mathcal{H C}}$. Since $A b s t_{\mathcal{W}}(\{\})=\{\}$, Proposition 1 ensures monotonicity of a sequence of $A D_{P}$ applications and we can define its ordinal powers in the usual way.

Theorem 3. $A D_{P}$ is finitary.

Proof. Suppose $A D_{P}$ is not finitary: There does not exist $n \in N$ such that $A D_{P} \uparrow n$ is a fixpoint. Since $A D_{P}$ is monotonic, it will then construct an infinite $D$. Now, for any $D \in \mathcal{D}_{\mathcal{H C}}$, Clauses $(D)$ and $T_{P}^{C}($ Clauses $(D))$ are both finite sets, thus in each iteration of $A D_{P}$, only a finite number of new clauses are added to $D$. The only way then in which $D$ can become infinite is through an infinite path in $D$. But this would mean there exists an infinite series of clauses $C_{1}, \ldots, C_{k}, \ldots$ without two clauses $C_{i}, C_{j}(i \leq j)$ such that $\left(C_{i}, C_{j}\right) \in \mathcal{W}$, contradicting well-quasiness of $\mathcal{W}$.

Corollary 2. Given a program $P$ and an abstraction function Abst W , the program $A D_{P} \uparrow n_{0} \cup \mathcal{R e s}\left(\right.$ Clauses $\left.\left(A D_{P} \uparrow n_{0}\right)\right)$, where $A D_{P} \uparrow n_{0}$ is the fixpoint of $A D_{P}$, is a bottom up partial deduction of $P$ (using $A b s t_{W}$ ).

Example 11. The bottom up partial deduction of $P$ (using $A b s t_{\mathcal{W}_{\triangleleft}}$ ) is the following program (where only the predicates of interest, append and reverse, are given)

append $([], L, L) \leftarrow$ append $([X], L,[X \mid L]) \leftarrow$

append $\left(\left[X \mid X_{s}\right], Y,[X \mid Z]\right) \leftarrow \operatorname{append}\left(X_{s}, Y, Z\right)$

$\operatorname{append}(X, Y, Z) \leftarrow \operatorname{append}(X, Y, Z)$ 


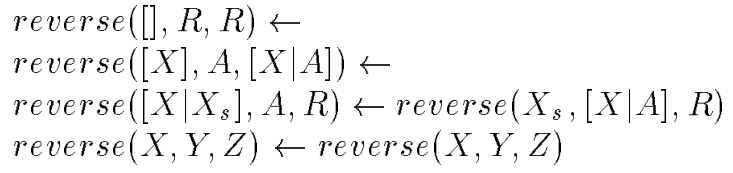

For both predicates, input lists of length 0 or 1 are captured by a unit clause. This is due to the fact that when Abst detects that

$$
\text { (reverse }([, R, R) \unlhd \text { reverse }([X], A,[X \mid A]))
$$

the latter is generalised (so called child generalisation in top down partial deduction terminology, see Figures 3 and 4). Generalising the former instead and removing its descendant graph - (parent generalisation) usually results in less generated code (in the example: only one unit clause, for the empty input list, would be generated). However, child generalisation is necessary if we want $A_{P}^{C}$ (and $A D_{P}$ ) to remain monotonic. On the other hand, when parent generalisation is used, a sequence of $A_{P}^{C}$ applications is no longer monotonic, but $A_{P}^{C}$ can still be finitary due to a "sufficiently large" monotonic subsequence. How to incorporate this exactly into the framework is a topic of further research. The concrete control strategy of [21] uses parent generalisation.

\section{Discussion \& Future Work}

This paper provides a formal framework for bottom up specialisation. We enrich the compositional $T_{P}^{C}$ operator $[2,1]$ with abstraction and thus obtain a generic bottom up partial deduction operator $A_{P}^{C}$. We prove soundness and completeness of the transformation provided a particular condition is imposed on $A_{P}^{C}$. This inside-closedness condition plays a key role in deciding whether actually to rename an atom or use the atom itself in the abstraction. Always renaming during abstraction creates a set $A_{P}^{C} \uparrow n_{0}$ which trivially is inside-closed, and hence the residual program derived from it is sound and complete. The clauses from $\mathcal{G} \operatorname{en}\left(A_{P}^{C} \uparrow n_{0}\right)$ serve as "translation" clauses between the renamings and the original predicates. On the other hand, never renaming during abstraction creates a set $A_{P}^{C} \uparrow n_{0}$ leading to a residual program $P^{\prime}$ that possibly is not sound with respect to $P$. Deriving $P^{\prime}$ from $P$ by the control strategy of [21] provides an example: if $A \in M_{s}\left(P^{\prime}\right)$, it is possible that $A \notin M_{s}(P)$; however $A$ is "correct" in the sense that there does exist an $A^{\prime} \in M_{s}(P)$ such that $A^{\prime} \preceq A$ (Theorem 3.16 in [21]). In general, clauses from $\mathcal{G} \boldsymbol{e n}\left(A_{P}^{C} \uparrow n_{0}\right)$ that bear the same predicate in head and body, can be filtered from the residual program since they do not serve as "translation" clauses. Herefore, less renaming results in smaller transformed programs. Examples 10 and 11 required none.

The aim of bottom up partial deduction should be to maximize the propagation of information in the program, while preserving the least S-Herbrand model. Without abstraction, the process reduces to bottom up evaluation and $P^{\prime}=M_{s}(P)$. Enriching $T_{P}^{C}$ with abstraction introduces several new complications. While in general $A_{P}^{C}$ is not continuous, it can be defined finitary. This is mandatory if we plan to use it for program transformation, since the transformation as well as the resulting program must be finite. Also, while the defined 
$A_{P}^{C}$ operator is monotonic, practical control (as the one in [21]) may use parentgeneralisation (cutting away parts of a DAG built so far), and destroy overall $A_{P}^{C}$ monotonicity.

As noted in [2,1], $T_{P}^{C} \uparrow \omega$ is in essence a set of resultants which can be seen as the result of a top down partial deduction of $P$ w.r.t. a set of atomic goals of the form $p\left(X_{1}, \ldots, X_{n}\right)$. As a consequence of abstraction, this will not be the case with our transformation. Reconsider the program of Example 2 to which the clause $q(Y) \leftarrow p(X, Y)$ is added. Abst is defined as in Example 3, resulting in a set $I_{2}$ which includes the clause $q(Y) \leftarrow p(a, Y)$. Although this clause was produced by $T_{P}^{C}$ starting from an abstraction, it can not be derived as a resultant from a top down partial deduction of $q(Y)$, since its body contains the constant $a$ as the result of an earlier bottom up propagation.

Recently, an integration between partial deduction and abstract interpretation (both top down) has been established [13]. A similar integration between our framework and bottom up abstract interpretation (see e.g. [3]) might be feasible and is a topic of further research. Also the exact relation between bottom up and top down partial deduction needs to be scrutinised, as well as an integration between the two techniques. Examples in [21] indicate that a combination of the described bottom up transformation with a top down component using a trivial control strategy - deterministic unfolding - is capable of specialising the Vanilla meta interpreter satisfactorily. Equally good results can be obtained by a top down control strategy alone, but often at the cost of not completely general and/or automatic techniques (e.g. [12]), or a non-trivial and complex control mechanism. In [20], a sophisticated approach is described based on extending a local unfolding rule by global information. Although the resulting technique is completely general and automatic, in spite of its complexity, it turns out to be not sufficiently effective when more involved meta interpreters are at hand. The same results can be obtained by two separate but rather straightforward control strategies, and the combined approach is conceptually cleaner than the sophisticated top down one. We plan to investigate how the two control strategies can be tuned - separately and combined - to obtain good specialisation of other, more involved meta interpreters.

\section{Acknowledgments}

We thank Karel De Vlaminck for his continuing support and interest in this work. We also thank anonymous referees for interesting and constructive comments.

\section{References}

1. A. Bossi, M. Gabbrielli, G. Levi, and M. Martelli. The S-semantics approach: Theory and applications. Journal of Logic Programming, 19/20:149-197, 1994.

2. A. Bossi, M. Gabbrielli, G. Levi, and M. C. Meo. A compositional semantics for logic programs. Theoretical Computer Science, 122(1-2):3-47, 1994. 
3. Michael Codish, Saumya K. Debray, and Roberto Giacobazzi. Compositional analysis of modular logic programs. In Conference Record of the Twentieth ACM SIGPLAN-SIGACT Symposium on Principles of Programming Languages, pages 451-464, Charleston, South Carolina, January 10-13, 1993. ACM Press.

4. Y. Cosmadopoulos, M. Sergot, and R. W. Southwick. Data-driven transformation of meta-interpreters: A sketch. In H. Boley and M. M. Richter, editors, Proceedings of the International Workshop on Processing Declarative Knowledge (PDK'91), volume 567 of $L N A I$, pages 301-308. Springer Verlag, 1991.

5. D. De Schreye, M. Leuschel, and B. Martens. Tutorial on program specialisation (abstract). In J.W. Lloyd, editor, Proceedings ILPS'95, pages 615-616, Portland, Oregon, December 1995. MIT Press.

6. F. Denis and J.P. Delahaye. Unfolding, procedural and fixpoint semantics of logic programs. In 8th Annual Symposium on Theoretical Aspects of Computer Science, volume 480 of LNCS, pages 511-522. Springer, 1991.

7. N. Dershowitz and J.-P. Jouannaud. Rewrite systems. In J. van Leeuwen, editor, Handbook of Theoretical Computer Science, Vol. B, pages 243-320. Elsevier, MIT Press, 1990.

8. M. Falaschi, G. Levi, M. Martelli, and C. Palamidessi. Declarative modeling of the operational behaviour of logic programs. Theoretical Computer Science, 69:289$318,1989$.

9. J. Gallagher. Specialisation of logic programs: A tutorial. In Proceedings PEPM'93, ACM SIGPLAN Symposium on Partial Evaluation and Semantics-Based Program Manipulation, pages 88-98, Copenhagen, June 1993. ACM Press.

10. R. Glück, J. Jørgensen, B. Martens, and M. H. Sørensen. Controlling conjunctive partial deduction of definite logic programs. In H. Kuchen and S.D. Swierstra, editors, Proceedings PLILP'96, pages 152-166, Aachen, Germany, September 1996. Springer-Verlag, LNCS 1140.

11. H. J. Komorowski. Synthesis of programs in the partial deduction framework. In Michael R. Lowry and Robert D. McCartney, editors, Automating Software Design, pages 377-403. AAAI Press, 1991.

12. A. Lakhotia and L. Sterling. How to control unfolding when specializing interpreters. New Generation Computing, 8(1):61-70, 1990.

13. M. Leuschel. Program specialisation and abstract interpretation reconciled. In J. Jaffar, editor, Proceedings of the Joint International Conference and Symposium on Logic Programming, Manchester, United Kingdom, June 1998. MIT-Press.

14. M. Leuschel and B. Martens. Global control for partial deduction through characteristic atoms and global trees. In O. Danvy, R. Glück, and P. Thiemann, editors, Proceedings Dagstuhl Seminar on Partial Evaluation, pages 263-283, Schloss Dagstuhl, Germany, 1996. Springer-Verlag, LNCS 1110.

15. M. Leuschel, B. Martens, and D. De Schreye. Controlling generalisation and polyvariance in partial deduction of normal logic programs. ACM Transactions on Programming Languages and Systems, 20(1), 1998.

16. J. W. Lloyd and J. C. Shepherdson. Partial evaluation in logic programming. Journal of Logic Programming, 11(3\&4):217-242, 1991.

17. B. Martens and J. Gallagher. Ensuring global termination of partial deduction while allowing flexible polyvariance. In L. Sterling, editor, Proceedings ICLP'95, pages 597-611, Shonan Village Center, Kanagawa, Japan, June 1995. MIT Press.

18. M. H. Sørensen and R. Glück. An algorithm of generalization in positive supercompilation. In J.W. Lloyd, editor, Proceedings ILPS'95, pages 465-479, Portland, Oregon, December 1995. MIT Press. 
19. W. Vanhoof. Bottom up information propagation for partial deduction. In M. Leuschel, editor, Proceedings of the International Workshop on Specialization of Declarative Programs and its Applications, pages 73 - 82, Port Jefferson, Long Island N.Y. (USA), October 1997. Proceedings available as report CW255, Dept. of Computer Science, K.U.Leuven, Belgium.

20. W. Vanhoof and B. Martens. To parse or not to parse. In N. E. Fuchs, editor, Proceedings of the Seventh International Workshop on Logic Program Synthesis and Transformation LOPSTR '97, volume 1463 of Lecture Notes in Computer Science, pages 314 - 333, Leuven, Belgium, 1997. Springer-Verlag.

21. W. Vanhoof, B. Martens, D. De Schreye, and K. De Vlaminck. Specialising the other way around. In J. Jaffar, editor, Proceedings of the Joint International Conference and Symposium on Logic Programming, Manchester, United Kingdom, June 1998. MIT-Press. 


\title{
A Figures
}

In this appendix, the Dags built in the examples throughout Section 4 are depicted. For simplicity, variables in the DAGs are not renamed.

\author{
list_nil([]) \\ list_notnil([X|Xs]) \\ list_head([X|Xs],X) \\ list_tail([X|Xs],Xs) \\ list_cons(X,Xs,[X|Xs])
}

Fig. 1. $D_{P} \uparrow 1=A D_{P} \uparrow 1$

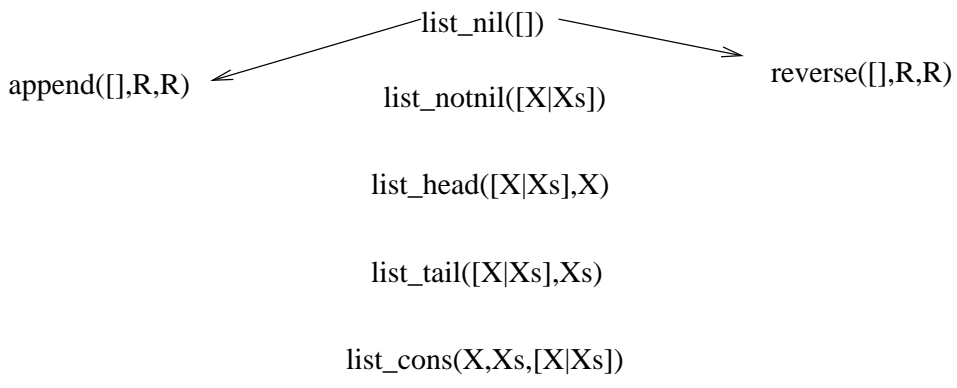

Fig. 2. $D_{P} \uparrow 2=A D_{P} \uparrow 2$

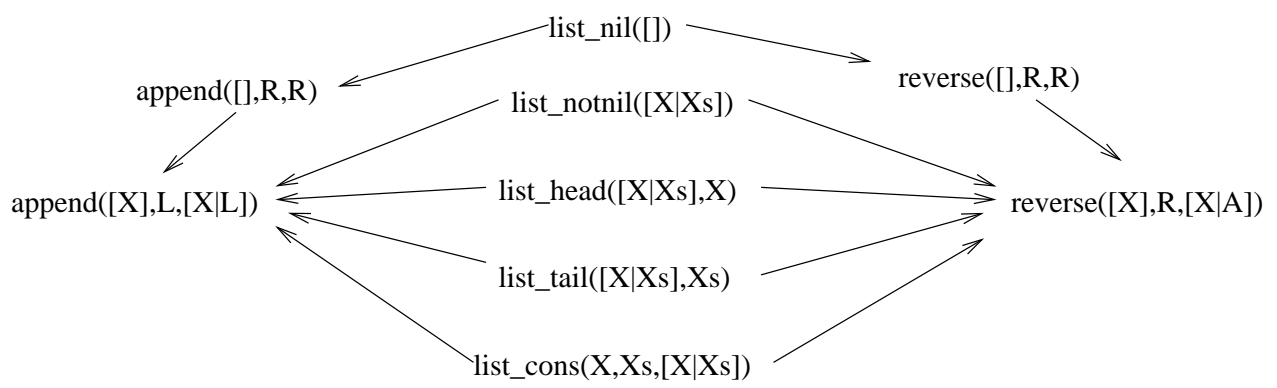

Fig. 3. $D_{P} \uparrow 3$ 


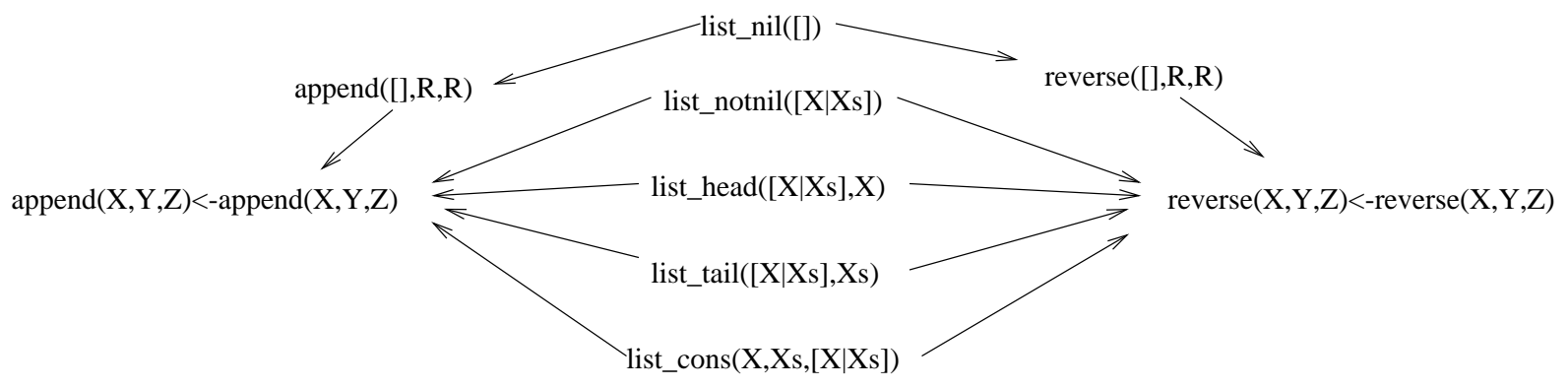

Fig. 4. $A D_{P} \uparrow 3$

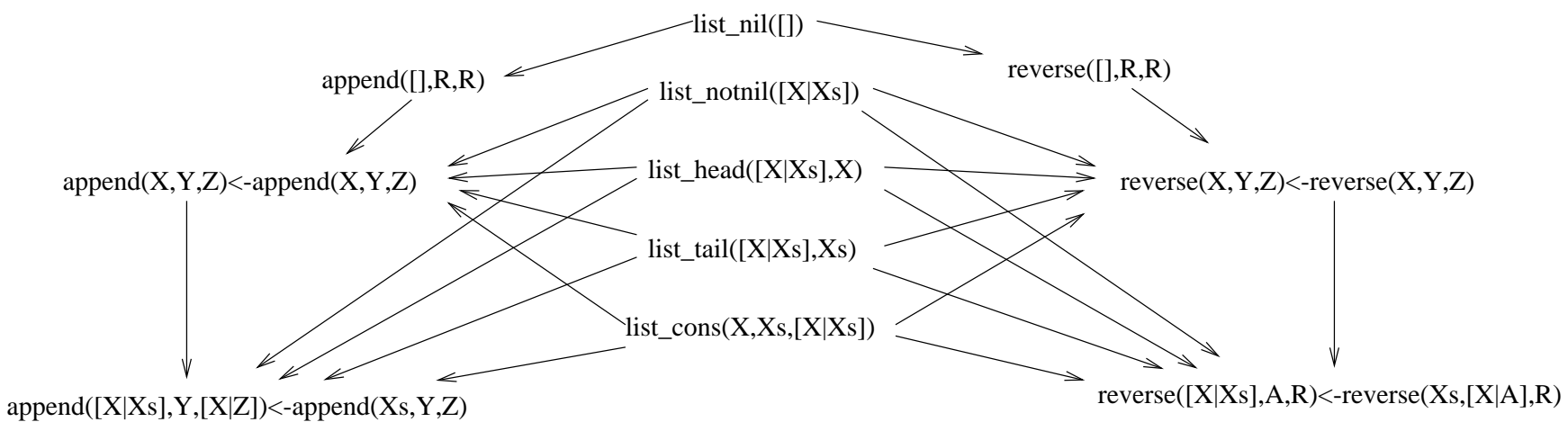

Fig. 5. $D_{P} \uparrow 4$

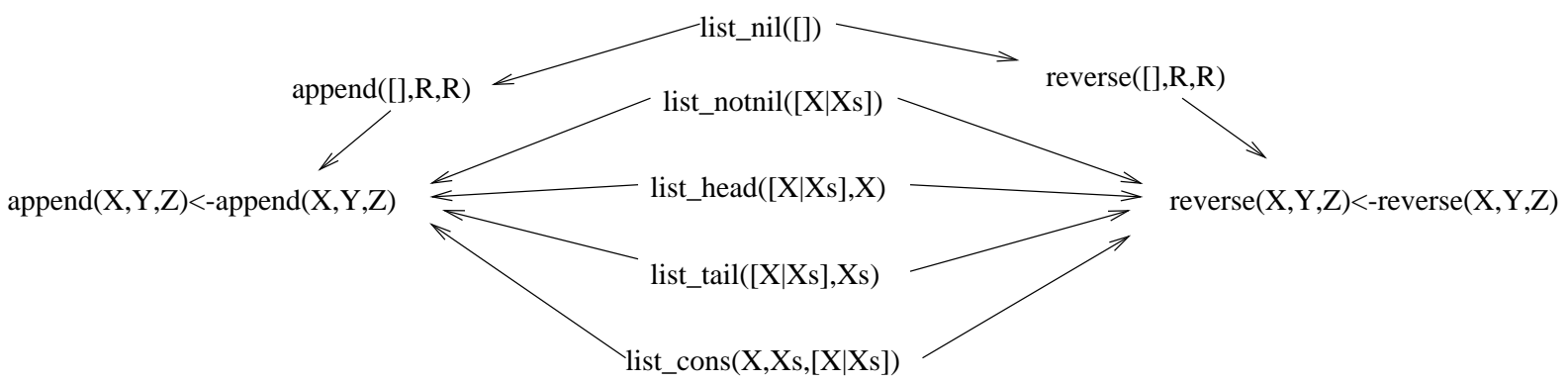

Fig. 6. $A D_{P} \uparrow 4=A D_{P} \uparrow 3$ 